Intersections

Canadian Journal of Music

Revue canadienne de musique
Intersections CANADIAN JOURAL OF MUSIC

\title{
Enseignement à distance de la musique ou l'e-learning musical
}

\section{Sylvaine Martin de Guise}

Volume 29, numéro 2, 2009

URI : https://id.erudit.org/iderudit/1000041ar

DOI : https://doi.org/10.7202/1000041ar

Aller au sommaire du numéro

\section{Éditeur(s)}

Canadian University Music Society / Société de musique des universités canadiennes

ISSN

1911-0146 (imprimé)

1918-512X (numérique)

Découvrir la revue

Citer cet article

Martin de Guise, S. (2009). Enseignement à distance de la musique ou

l'e-learning musical. Intersections, 29(2), 84-108.

https://doi.org/10.7202/1000041ar

\section{Résumé de l'article}

Aujourd'hui l'e-learning musical est un outil d'apprentissage de la musique en plein essor depuis le développement des technologies tel que le standard MIDI - permettant la communication entre ordinateurs et instruments de musique -, Internet, le Web et les multimédias. Tous ces domaines reliés favorisent la création de cours mis sur logiciels qui peuvent être diffusés sur Internet ou en Intranet dans les écoles. Cette avancée dans l'éducation musicale se constate partout dans le monde et nous verrons dans cet article différents exemples et procédés d'apprentissage à distance de la musique qui sont mis en oeuvre en France et au Canada.
All Rights Reserved (C Canadian University Music Society / Société de musique des universités canadiennes, 2010
Ce document est protégé par la loi sur le droit d'auteur. L'utilisation des services d'Érudit (y compris la reproduction) est assujettie à sa politique d'utilisation que vous pouvez consulter en ligne.

https://apropos.erudit.org/fr/usagers/politique-dutilisation/ 


\title{
CHRONIQUES : \\ ENSEIGNEMENT À DISTANCE DE LA MUSIQUE OU L'E-LEARNING MUSICAL
}

\author{
Sylvaine Martin de Guise
}

\section{INTRODUCTION}

Actuellement, la formation à distance par Internet, ou l'e-learning, s'étend à toutes les disciplines de l'éducation. L'enseignement de la musique n'échappe pas à la tendance générale grâce au protocole de communication MIDI $^{1}$ qui permet le dialogue entre machines et instruments de musique traditionnels et la transformation en données informatiques des éléments fondamentaux musicaux : hauteur de sons, durée, volume, timbre, transcription. Toutes ces possibilités ouvrent des voies inédites pour l'enseignement de la musique qui emploient les nouvelles technologies, associées au réseau Internet et au système hypertexte.

Les TICCE$^{2}$ (Technologies de l'Information, de la Communication et de la Création pour l'Enseignement) veulent répondre aux exigences et aux critères de l'enseignement musical en proposant un éventail d'outils multimédia (vidéos, enregistrements sonores, copies de partition, plateformes de communication) qui peuvent appuyer et renouveler le travail traditionnel d'un professeur de musique. Afin d'observer le progrès des cours à distance de la musique dans les systèmes scolaires (écoles primaires et secondaires, cégeps ou lycées) et les institutions spécialisées (écoles de musique, conservatoires et facultés de musique des universités), il faut faire un bref récapitulatif du formidable développement des technologies de l'audiovisuel et de l'informatique depuis leurs origines jusqu’au début du XXI siècle.

\section{DÉVELOPPEMENT PARALLÈLE ET CONCOMITANT DE L'AUDIOVI- SUEL ET DE L'INFORMATIQUE}

La formation à distance et l'e-learning qui s'exercent de nos jours dans toutes les sphères de l'éducation représentent l'aboutissement de la notion d'enseignement à distance telle qu'elle a été imaginée la première fois, aux débuts de la radio (1929) et de la télévision (1949). L’e-learning musical, ou l'apprentissage musical par Internet, en est un des ultimes développements. Cependant, il faut rappeler que l'idée de l'enseignement à distance de la musique est avant tout née

\footnotetext{
1 MIDI est l'acronyme de Musical Instrument Digital Interface.

2 Michel Bézard, "TICCE : le MIDI à l'heure du second C ", Les dossiers de l'ingénierie éducative - Des outils pour la musique, $\mathrm{N}_{43}^{\circ}$ (juin 2003):1.
} 
de l'émergence d'une technologie : la réalisation des premiers enregistrements sur disque vinyle (1950). En effet, la captation sonore de la musique sur un objet, un microsillon ou une bande enregistrée, a permis d'en analyser le contenu et de partager ce contenu de manière inédite, bref de concevoir désormais une méthode didactique innovatrice portant sur l'étude de la musique. C'est ainsi que, dès l'émergence de ces nouveaux moyens de communication, on a soupçonné et compris l'énorme potentiel pédagogique qu'ils recélaient indépendamment des domaines que ces moyens de communication pouvaient couvrir et de la façon dont ils pouvaient se décliner à leur abord. Parallèlement, on a assisté à la progression de l'informatique (1930), à la fabrication des premiers ordinateurs (1940) et à la création du disque compact (1978) pouvant stocker des données sous forme numérique destinées à être gérées par les ordinateurs. Les années 1980 débutent avec l'arrivée des microordinateurs et se terminent avec l'apparition du multimédia. Ce dernier mot désigne des applications rendues possibles par la combinaison de la mémoire de l'ordinateur, du disque compact (CD) et des fonctions de l'ordinateur. Ces applications, ou logiciels, permettent la gestion, l'utilisation et la direction de différents médias simultanément : musique, son, image, vidéo et interface homme-machine. Les progrès techniques et le faible coût des mémoires et des réseaux font que la technologie multimédia, voire hypermédia ${ }^{3}$, est maintenant disponible avec beaucoup de facilité. Ces découvertes audiovisuelles et informatiques ont eu des retombées directes, à des degrés divers, dans la pratique des musiciens, toutes spécialités confondues. Compositeurs, interprètes, professeurs, chefs d'orchestre, musicologues, producteurs, éditeurs, tous ont commencé à s'intéresser, même s'ils les dédaignaient, à ces nouvelles ressources et possibilités d'expression de la musique.

\section{LES TICE ET LES TICCE}

De nos jours, la formation à distance, que ce soit de la musique ou de tout autre domaine, signifie associer les moyens technologiques modernes aux méthodes d'enseignement classique, ce qui ouvre le champ des TICE (Technologies de l'Information et de la Communication pour l'Éducation). Ces technologies, qui sont appelées communément les TICE pour tous les domaines de l'éducation, deviennent TICCE pour les domaines de l'éducation artistique. En effet, le « $\mathrm{C}$ » ajouté représente la part de création qui n'est pas absolument exigée dans l'esprit des autres disciplines. Les TICE et les TICCE offrent au public un support pédagogique original en utilisant des procédés techniques développés en multimédia pour rénover les systèmes d'éducation habituels et contribuer ainsi à leur modernisation. Ces technologies engagent automatiquement un effort de normalisation et de standardisation de leurs procédés et de leurs dif-

3 Hypermédia vient de "hypertexte » qui est un système contenant des documents liés entre eux par des hyperliens permettant de passer automatiquement (en pratique grâce à l'informatique) du document consulté à un autre document lié. Un document hypertexte est donc un document qui contient des hyperliens. Lorsque les documents ne sont pas uniquement textuels, mais aussi audiovisuels, on peut parler de système et de documents hypermédias. 
fusions : c'est ici qu'interviennent, dans les modèles (logiciels) de cours, les normes et les standards tels que LOM 4 , SCORM 5 , MPEG-21 $1^{6}$, etc. Les TICCE ont une implication particulière dans la musique parce que l'enseignement de cet art suppose que l'on puisse non seulement l'expliquer par des textes ou des partitions, mais que l'on puisse le faire entendre en appuyant chacune des illustrations visuelles par une illustration sonore. Enfin, il s'agit aussi de pouvoir reproduire ou créer des œuvres musicales originales avec ces mêmes moyens des TICCE.

\section{I- DÉVELOPPEMENT DE L'E-LEARNING MUSICAL Les débuts}

On sait que l'enseignement musical «à distance » a été initié bien avant Internet : il a ses précurseurs et ses archétypes qui sont les programmes culturels à la radio et à la télévision, et l'université à la télévision?. Grâce à ces initiatives, une tradition est déjà inscrite : elle est approfondie et devenue incontournable depuis les années 1970 avec les enregistrements musicaux de grande qualité rendus possibles par l'enregistrement analogique sur cassette puis, au début des années 1980, par l'enregistrement numérique sur CD. Dans la perspective d'un enseignement de la musique de bonne tenue, les outils d'enregistrements sonores et musicaux ont été enrichis avec succès par la généralisation des techniques multimédias, de l'usage des ordinateurs et du protocole de communication MIDI, comme ce fut le cas, dès 1995, des essais d'interopérabilité de ce protocole avec d'autres normes ou spécifications, telles que SCORM (pédagogie) et MPEG (audiovisuel). Ainsi, dans les années 1990, l'on assiste à la naissance du « home studio » qui autorise tout un chacun, avec un investissement modéré, de réaliser d'excellents enregistrements et de graver des CDs sur un ordinateur personnel. Cela donne une grande liberté d'action et la possibilité de créer des petits modules d'enseignement sur logiciels de la musique qui sont quasi autonomes et qui donnent satisfaction, tant aux maîtres qu'aux élèves. Dès lors, dans toutes les facultés de musique des universités, les conservatoires ou les écoles d'enseignement de la musique, nous trouvons des professeurs qui conçoivent leurs cours en utilisant ces nouvelles technologies. De même, les enseignants peuvent eux-mêmes créer des exercices de musique sur des supports analogiques ou numériques (les bandes ou les cassettes d'enregistrements audio) mis à la disposition des étudiants, qu'ils pourront pratiquer chez eux ou dans une classe aménagée à cet effet. Bien qu'à l'origine expérimental, person-

4 LOM (Learning Object Metadata) est un modèle de description de données pour des objets d'apprentissage.

5 SCORM (Sharable Content Object Reference Model) est une spécification permettant de créer des objets pédagogiques structurés.

6 MPEG-21 (Moving Picture Experts Group) est une norme proposant une architecture pour l'interopérabilité et l'utilisation simple de tous les contenus multimédias.

7 L'enseignement télévisuel est actif depuis longtemps dans les universités dans le domaine de la formation continue qui s'adresse aux professionnels. Quelques exemples : en France, Centre de Télé-Enseignement Universitaires (CTEU), les Services d'Enseignement à Distance (SEAD) ; au Canada, au Québec, Télé université du Québec (Supérieur) ; en Suisse, Formation universitaire à distance (Supérieur), etc. 
nel et marginal, cet emploi des technologies dans l'enseignement musical est devenu bientôt tellement répandu et fréquent qu'il s'est finalement fait remarquer par les instances et les autorités de l'éducation et de la pédagogie. Voyons ce qui s'est passé en France.

\section{Enseignement à distance de la musique pratiqué dans les écoles françaises}

En France, le Ministère de l'éducation a rapidement été informé de ce phénomène de " création de cours de musiques par l'informatique " et s'est intéressé dès 1992 à s'associer aux grandes institutions de recherches musicales comme l'IRCAM (Institut de Recherche et Coordination Acoustique/Musique), le GRM (Groupe de Recherches Musicales) et MIM (Musique et Informatique de Marseille) afin de produire des contenus éducatifs de musique pouvant convenir au système scolaire français, de l'élémentaire au lycée (Maestracci 2003). La réussite est exemplaire.

Depuis 1998, dans le cadre de ses missions pour développer l'usage des technologies de l'information et de la communication dans l'éducation le Ministère mène une politique de développement de ressources multimédias pédagogiques, qui se traduit notamment par un dispositif de soutien élaboré et mis en œuvre par la Direction de la technologie. (Plas 2003, p. 42)

Plusieurs outils ont été mis en place; ils existaient déjà commercialement ou ont été développés avec la demande académique. Ils sont énumérés ici indifféremment dans leur ordre d'apparition dans l'usage commercial et dans le système public d'enseignement.

Étienne Gégout, professeur d'éducation musicale, coordonnateur du réseau TICE Éducation Musicale, Direction de la technologie (Gégout 2003, p. 46-47), décrit le « kit » indispensable au professeur d'éducation musicale en sept classes d'outils ; la $8^{\mathrm{e}}$ classe (le cartable électronique) est ajoutée par Jean-Marc Maurer (2003, p. 38) :

1. Les outils de création au service des cuvres musicales (par exemple, MusicLab) : l'outil de création Musique Lab - conçu pour l'Éducation nationale par des professeurs en collaboration avec l'IRCAM - est une série logicielle composée de six modules (Hauteur et intensité, Polycycles, Construction rythmique, Échelles et modes, Nuages, Montage). MusiqueLab permet l'étude, la manipulation et l'expérimentation des notions essentielles du langage musical qui organisent toutes les esthétiques et proposent des ateliers de création sonore.

2. Les séquenceurs MIDI (par exemple, Cubase) : un séquenceur MIDI est un appareil ou logiciel qui permet de gérer des données MIDI et qui possède des fonctions d'enregistrement sur plusieurs canaux virtuels. Les marques connues sont Logic Pro, Cubase (de marque RIP - Reconnu d'Intérêt Pédagogique) et Cakewalk.

3. Les éditeurs de partition (par exemple, Finale) : un logiciel éditeur de partitions sert à retranscrire des musiques sur des portées musicales. Il 
est souvent intégré dans le séquenceur lui-même, ou «brut » comme les marques Finale, Encore et Sibelius.

4. Les éditeurs audionumériques (par exemple, Sound Forge) : l'éditeur audionumérique est un logiciel qui permet d'enregistrer, de corriger, de transformer et de monter le son qu'on enregistre ou qui sont préenregistrés. On connaît les marques Sound Forge, WaveLab, CoolEdit.

5. Les " arrangeurs" (par exemple, Band In Box) : un arrangeur est un logiciel qui permet de transformer un enchaînement harmonique en un accompagnement instrumental dans le style choisi par l'utilisateur. La marque la plus connue est Band in Box.

6. Les logiciels de présentation (par exemple, Asymétrix Présentation) : un logiciel de présentation permet de créer des applications multimédia qui manipulent des fichiers MIDI, sonores et vidéo. Le logiciel le plus apprécié des professeurs de musique est Asymétrix Présentation.

7. Les bancs de montage multimédia (par exemple, Music Maker) : un banc de montage ou une plateforme de montage multimédia est un logiciel qui permet de traiter simultanément des images, des sons MIDI, Wave et $\mathrm{MP}_{3}$ et des vidéos. La marque la plus utilisée dans l'éducation nationale est MusicMaker.

8. Le cartable électronique : le cartable électronique est une dénomination de l'ensemble des outils réunis - ordinateur portable, Internet et logiciels - qui est mis à la disposition de l'étudiant.

\section{Cohabitation de deux approches pédagogiques : l'autoformation et la for- mation traditionnelle}

\section{Définition de l' "autoformation":}

Avec l'implication des TICE et des TICCE dans l'enseignement, nous découvrons une nouvelle approche pédagogique, "l'autoformation ", qui s'ajoute à la première, «la formation traditionnelle ». L'autoformation, que nous aurions appelée autrefois autodidaxie, consiste à encourager l'étudiant à pratiquer une grande autonomie dans ses acquisitions tout au long de son apprentissage. En effet, l'e-learning part du principe qu'il met à la disposition de l'étudiant tous les outils et les moyens de la formation qu'il a choisie. Le dispositif, qui se veut très complet et souple, est d'habitude amplement expliqué au futur étudiant, qu'on appelle désormais " apprenant » en langage d'apprentissage à distance. Ce dispositif est ainsi composé de séances en "présentiel ». Ceci signifie qu’une rencontre classique entre professeur et étudiant(s), à une date et à une heure fixe, est organisée. Ces séances sont suivies de sessions en « distanciel » : elles offrent à l'apprenant la possibilité de travailler à un contenu de cours mis en ligne dans un site sur Internet selon ses propres disponibilités, dans le temps et dans le lieu de son choix (le soir, le week-end, chez lui ou à l'étranger...). Enfin, l'apprenant peut rester en contact avec un " tuteur", variation du traditionnel assistant du professeur, et en relation avec ses collègues étudiants par le biais de forums et de rencontres en visioconférences.

Dans une formation générale, l'e-learning n'est pas le seul mode d'enseignement recommandé, surtout dans l'apprentissage du musicien professionnel, 
qu'il soit compositeur, interprète ou musicologue. En effet, l'e-learning musical doit s'ajouter aux outils d'apprentissage conventionnels et convenus pour constituer avec ces derniers une version moderne du métier de musicien. En effet, l'expérience nous montre que les formations spécialisées, en musique comme d'autres disciplines, continuent d'exiger de suivre les grandes lignes de l'enseignement traditionnel comportant la rigueur indispensable à une formation de qualité. Dans ces institutions, la hiérarchie des spécialisations et l'organisation des matières ont, de tout temps, été réactualisées : les contenus des cours sont régulièrement adaptés aux découvertes et aux méthodes du moment et, finalement, l'absolue nécessité du contact avec des maîtres de renom ou de la régie par de grands directeurs, reste incontournable, outre les progrès technologiques. Aussi, lorsque l'on souhaite adapter à distance des cours des écoles musicales professionnelles, il semble préférable de se conformer dans un premier temps à la forme classique du cours pour que la valeur ajoutée que représente l'apport technologique puisse être intégrée de façon pertinente en développant des supports d'éducation inédits. On peut employer l'e-learning en respectant les impératifs de l'éthique professionnelle dans l'enseignement de la musique, comme en observant les obligations sociales et économiques de ces institutions. Depuis longtemps, les progrès technologiques multimédias et audiovisuels sont utilisés pour être mis au service de ce savoir héréditaire et séculaire que représente la pratique musicale occidentale. L'e-learning peut être joint sans difficulté à la panoplie existante.

\section{Distinction entre les cours d' " autoformation " et les cours "traditionnels mis à distance " :}

Pour la clarté de l'exposé, il est approprié de distinguer les deux types de cours à distance suivants :

1. Les cours de type " autoformation ", (sur logiciels) que nous trouvons déjà en très grand nombre dans toutes les universités et les écoles de musique dans le monde.

2. Les cours de type "traditionnels", calqués sur le modèle des matières enseignées dans les facultés de musique et des classes des conservatoires, qui sont mis à distance (sur vidéos, PDF, etc.) et de quelle façon ils sont réalisés.

Sont ici détaillés les cours d'autoformation, en profitant des exemples cités correspondants, pour voir :

a) La question des moyens techniques employés dans ces cours - qui sont sur logiciels, sur vidéos ou entièrement sous formats PDF - et qui utilisent à des degrés divers des fichiers sonores $\mathrm{MP}_{3}$ et des fichiers d'images JPEG, des animations Flash, etc.

b) La question des droits d'auteur, d'exécution et de reproduction.

\section{II- LES COURS D'AUTOFORMATION}

Il existe aujourd'hui autant de façon d'utiliser les nouvelles technologies dans un cours de musique qu'il y a de sortes de cours, voire de professeurs qui enseignent la musique et qui créent dès lors un produit original, marqué de leur 
personnalité. Souvent un enseignant qui décide d'employer des ressources technologiques modernes pour montrer différemment ses leçons et supporter autrement son programme est un éducateur qui n'hésite pas à sortir des sentiers battus. Pour ce professeur, il s'agit d'une création personnelle et d'une " œuvre " pédagogique. Il est à l'écoute de ce qui fascine et environne naturellement ses élèves d'aujourd'hui, et veut tenter de les rejoindre ou de les sensibiliser à la matière ou la discipline musicale qu'il enseigne par l'utilisation d'outils de communication connus et largement répandus auprès de ce jeune public - Internet, le chat, les lecteurs $\mathrm{MP}_{3}$, etc. Cette sorte d'enseignant sera donc notre spécialiste type des cours d'autoformation.

\section{Les moyens techniques 1) : les cours sur logiciels}

Les cours sur logiciels représentent le cours d'autoformation à la fois le plus répandu et le plus exigent au niveau de sa fabrication. En effet, ils demandent à l'éducateur une certaine connaissance des logiciels et des applications multimédia, tels que la technologie Flash, ou d'autres formats ou technologies. Ces cours sont généralement conçus pour permettre aux étudiants de pratiquer des exercices de dictées musicales, de formation de l'oreille ou d'analyses musicales. Par exemple, il s'agit pour l'apprenant de reconnaître des extraits des musiques (formation de l'oreille), de distinguer des parties des formes musicales des morceaux de musique (analyse musicale) ou de donner le nom des notes des musiques qui sont jouées (dictée musicale), etc. Ces exercices (qui peuvent être créés en très grand nombre une fois que le procédé technique est assimilé par le professeur) ont l'avantage de permettre une pratique abondante, voire infinie des mêmes exercices musicaux jusqu'à ce que l'étudiant maîtrise le sujet. Parmi les cours actuellement mis à distance, les cours de reconnaissance auditive sont ceux que les institutions et les professeurs adaptent volontiers sur logiciel, car il s'agit d'une matière qui nécessite le plus d'explications et de répétitions afin que l'étudiant soit à l'aise avec ses principes. Ces cours sont déjà en place à l'Université Laval à Québec et à l'Université Paris 8 à Paris.

\section{Exerciseurs de formation auditive (Faculté de musique de l'Université Laval à Québec)}

La Faculté de musique de l'Université Laval à Québec propose en ligne des « Exerciseurs de formation auditive " dont on peut faire l'essai gratuitement pendant quelques jours. Ensuite il est demandé de s'inscrire si on veut continuer de pratiquer cette formation.

\section{Logiciels de pratique de formation auditive (Faculté de musique de l'Univer- sité de Paris 8)}

La Faculté de musique de l'Université Paris 8 présente à ses étudiants des logiciels de pratique de dictées, de reconnaissances d'accords, etc. Ces derniers ne sont pas en ligne car les professeurs ont souvent employé de courts extraits de musiques connues sans avoir acquis les droits d'exécution ou de reproduction des morceaux. Néanmoins, ces logiciels sont mis à la disposition des étudiants 
dans les locaux de la Faculté sur des postes d'ordinateurs et leur utilisation ne sortant pas cadre de l'université, la question des droits est négligée et temporairement éludée. À dire vrai, on ferme les yeux sur ces pratiques car elles sont en total désaccord avec la loi française.

De fait, il existe en France «le droit de citation » qui concerne les images (ici, les partitions) et qui se rapproche de la doctrine du fair use (ensemble des règles de droit d'origine législative et jurisprudentielle) américain et canadien, en vigueur dans la plupart des pays de common law. On traduit en français par " usage loyal ", " usage raisonnable " ou " usage acceptable ». Mais on aura remarqué que le "droit de citation » s'étend aux images mais non pas aux " sons et à la musique ». D'où une extension du "droit de citation » aucunement légale en réalité. Mais nous revenons plus loin dans l'article, et à chaque fois que c'est nécessaire, sur la question des différents droits (d'auteur, d'exécution et de reproduction).

\section{Les moyens techniques 2) : les cours enregistrés entièrement sur vidéos}

Un autre type d'enseignement est la captation du cours sur vidéo : le cours entier est enregistré et l'enregistrement est configuré dans un format efficace pour la transmission par Internet. L'étudiant peut à sa guise arrêter le film du cours, le réécouter, le reprendre autant de fois qu'il en éprouve le besoin. Le cours sur vidéo peut être accompagné de sa version " papier » (PDF) que prépare le professeur à l'intention de l'étudiant de la même manière qu'un contenu de cours remis à l'étudiant qui vient en classe.

\section{Formation auditive FMA-113-08 et FMA-114-o8 (Conservatoire de musique du Québec à Rimouski)}

Josée April, et l'auteure du présent article, ont réalisé l'enregistrement d'un cours " live » de formation auditive à l'intention des étudiants du Conservatoire de musique du Québec à Rimouski. Ce cours a été enregistré sur vidéo et réalisé en cours à distance avec le modèle Opale de Scenari ${ }^{8}$. Force est de constater que les deux procédés, « logiciel ou exerciseurs » ou « live en vidéo » fonctionnent tous les deux très bien pour ce type de formation qu'est l'écriture musicale.

\section{Les moyens techniques 3 ) : les cours combinant les fichiers PDF, $\mathrm{MP}_{3}$ et JPEG}

À l'instar des cours dans d'autres domaines de l'éducation, on voit le cours sur " papier » qui est un résumé complet de la matière, distribué par email sur ordinateur et téléchargeable sous format PDF. En musique on ajoute souvent aux cours qui sont donnés en textes explicatifs et partitions les illustrations sonores sous forme de fichiers audio $\mathrm{MP}_{3}$ et des images sous forme de fichiers JPEG.

8 Opale est une chaîne éditoriale qui permet la rédaction, la gestion et la publication multisupport de documents de formation de type académique. Scenari est une suite logicielle libre de conception et d'utilisation de chaînes éditoriales pour la création de documents multimédia. 


\section{III- LE PROBLÈME DES DROITS D'AUTEURS, DE COMPOSITEURS, D'EXÉCUTION ET DE REPRODUCTION}

Il est courant de faire des photocopies des partitions dans le cadre des cours de musique des institutions d'enseignement et cette pratique est tolérée parce qu'elle ne sort pas de la classe et donc du cercle pédagogique. Il en va de même de l'échange en classe, ou du commerce sans but lucratif, des extraits d'enregistrements de musique : ils sont relativement consentis dans la mesure de l'exercice pédagogique. Cependant, juridiquement, cette question n'est pas exactement perçue de la même façon de part et d'autre de l'Atlantique ; les Français ont tendance à s'arrêter " au strict cercle de famille ", alors que la jurisprudence anglo-saxonne prend volontiers en compte la notion de fair use, citée plus tôt, pour les usages académiques ou en direction des handicapés. Cette question des droits d'auteurs et d'exécutions a longtemps fait obstacle à la diffusion et au progrès des cours à distance dans les établissements d'enseignement de la musique : c'est l'un des problèmes qui gêne l'avancement de l'e-learning musical. Toutefois, plusieurs solutions ont été trouvées, le plus souvent au cas par cas, et la décision finale de relever ce défi semble appartenir désormais à l'institution et aux professeurs qui acceptent de tenter l'expérience de l'enseignement en ligne ou à distance. Des outils ont été mis en place pour dégager une ligne de conduite et une méthode afin de résoudre la problématique des droits d'auteurs et compositeurs, mais les droits d'exécution et de reproduction constituent finalement les principales difficultés en ce qui concerne les formations « à distance » diffusées sur Internet.

\section{Droits d'exécutions : les différentes solutions possibles}

Lors d'une rencontre d'experts en science de la communication et de musiciens à la Maison des Sciences de l'Homme de Paris Nord concernant l'enseignement à distance de la musique, la question des droits d'auteurs et d'exécutions a été le premier sujet abordé. Étaient réunis à cette occasion Nicolas Desjardins ${ }^{9}$, Henri Hudrisier ${ }^{10}$, Guillaume Loizillon ${ }^{11}$, Gabriel Popovici ${ }^{12}$ et Priscilla Velut ${ }^{13}$.

\section{Contrat préalable}

Dans cette réunion, on a examiné la difficulté qu'il y a désormais d'exercer un contrôle sur la diffusion des enregistrements commerciaux des musiques qui concernent directement les droits que perçoivent les interprètes sur leurs enregistrements des œuvres musicales : droits d'exécution. Ainsi, l'idée a été suggérée d'établir et de payer un prix forfaitaire qui serait accordé au musicieninterprète, dès l'enregistrement d'une œuvre, en prévision de toutes les cir-

9 Nicolas Desjardins est Directeur général des Conservatoires d'art dramatique et de musique du Québec.

10 Henri Hudrisier est Maître de Conférence et HDR (Habilité à diriger des recherches) à l'Université de Paris 8.

11 Guillaume Loizillon, Professeur au Département de musique de l'Université de Paris 8.

12 Gabriel Popovici, Responsable du Centre de documentation de la Maison des Sciences de l'Homme Paris-Nord.

13 Priscilla Velut, Professeur en informatique de l'Université de Technologies de Compiègne. 
constances où ensuite cet enregistrement pourrait être utilisé. C'est un moyen simple qui a l'avantage d'être parfaitement réalisable et acceptable.

\section{Compilation spéciale faite par la maison de disque pour un établissement d'enseignement}

Concernant les cours d'autoformation que les professeurs ont déjà réalisés, des enregistrements de musique ont été utilisés sans en avoir acquis des droits d'exécution : il a été suggéré alors de séparer le contenu pédagogique de son contenu d'" exemples ». Ce qui veut dire que l'enseignant peut concevoir un cours comme une grille d'analyse qui est posée directement sur la source d'extraits musicaux. Et inversement, on peut décider de constituer un cours et de l'« habiller » ensuite de musiques sélectionnées avec l'accord et selon l'arrangement de la maison de disque - qui peut choisir de produire de son côté une édition de compilations spéciale en vue d'un enseignement déterminé ou de l'édition d'une compilation d'une grande variété d'extraits. Les étudiants n'ont plus qu'à se procurer l'enregistrement des extraits sonores comme ils achètent un livre de classe ou un manuel scolaire en début d'année.

\section{Dissociation du contenu pédagogique de son contenu d'exemples}

La dissociation du contenu du cours de ses exemples est une manœuvre complètement inédite mais absolument exécutable avec les modèles de chaînes éditoriales puisque leurs ingénieurs ont poussé à l'extrême l'idée de la séparation $d u$ fond et de la forme. Il faut simplement que l'enseignant exploite cette perspective qui lui est donnée au travers de l'emploi des chaînes éditoriales.

\section{Droits de reproduction : un constat fait par Serge Lacasse, professeur de la Faculté de musique de l'Université Laval à Québec}

Au Canada, par exemple, d'un point de vue strictement légal, pour qu'une musique originale puisse être diffusée en ligne, elle doit, au préalable, être fixée sur un fichier $\mathrm{MP}_{3}$, accessible sur un serveur, à partir desquels, pour être diffusée sur Internet, la SODRAC ${ }^{14}$ exige 500 \$ par « chanson » (soit une musique d'une durée d'environ 3 minutes) avant d'accorder les droits de reproduction dans le cadre d'un cours en ligne. Vu le coût de l'entreprise, Serge Lacasse conseille "d'exploiter les outils existants légaux (ex. iTunes, YouTube, Deezer ou MusicMe) pour faire entendre des extraits dans le cadre d'un cours en ligne parce que ces sites offrent la possibilité de créer des listes d'écoute " (Lacasse 2010), comme un étudiant se procurerait une liste de livres conseillée par un professeur afin de suivre son cours.

Nous terminons ici cette courte analyse des cours d'autoformation et des questions de droits qu'ils soulèvent pour aborder maintenant les cours traditionnels « mis à distance ».

14 Société canadienne de gestion du droit de reproduction qui représente les auteurs, compositeurs et éditeurs au Canada. 


\section{IV- COURS TRADITIONNELS MIS À DISTANCE}

Il existe différentes leçons de musique composées selon le domaine musical étudié. Cela va de l'apprentissage d'un instrument ou de la voix, en passant par la classe d'instrumentation ou d'orchestration, au cours d'histoire de la musique ; le premier se caractérise par un enseignement individuel, "du maittre à l'élève ", alors que le dernier peut être donné devant un très grand groupe. Entre les deux se présente un grand éventail de classes de cours, en fonction de la matière qu'ils traitent et du nombre d'élèves à qui ils peuvent s'adresser simultanément pour être bien expliqués et bien compris (de petits, moyens ou grands groupes de participants). Pour chacun des types de cours, nous donnerons un exemple de "réalisation à distance » qui sera commenté et explicité ensuite. Mais disons déjà qu'il y a "le cours privé » ou " en petit groupe », le " cours en moyen groupe » et le " cours magistral».

Le cours privé ou le cours en petit groupe représente la situation où un maître enseigne l'interprétation d'un instrument, comme, par exemple, la leçon de piano. Il peut durer une heure, ou davantage, et il existe la version en master class où le maître enseigne à l'élève devant les autres participants qui profitent de son exemple. Le cas est similaire avec le cours de composition qui peut se donner individuellement ou en petit groupe d'étudiants pour que chacun puisse profiter du travail de ses collègues.

- Ex. Cours de piano à distance du département de musique de l'Université d'Ottawa.

- Ex. Cours de saxophone donné par Benoît Plourde, Directeur du Conservatoire de musique du Québec à Rimouski.

Le cours en groupe moyen peut concerner, par exemple, le cours d'écriture musicale, harmonie tonale ou contrepoint, qui est donné généralement dans une petite classe d'un nombre limité d'élèves (une dizaine), et les cours théoriques et pratiques - de solfège, théorie et dictées musicales - qui peuvent regrouper une douzaine d'étudiants.

- Ex. Cours d'harmonie tonale de Josée April, professeur au Conservatoire de musique du Québec à Rimouski.

Il existe aussi les cours d'instrumentation ou d'orchestration, les classes d'analyse musicale et les classes d'ethnomusicologie, qui peuvent se pratiquer dans une classe moyenne d'une quinzaine d'étudiants ou plus.

- Ex. Cours d'ethnomusicologie de Monique Desroches, professeur d'ethnomusicologie de la Faculté de musique de l'Université de Montréal.

Enfin il y a le cours magistral, comme, par exemple, celui d'histoire de la musique, ou tout exposé de type " conférence ", qui est présenté dans un amphithéâtre et qui peut s'adresser à un grand groupe d'étudiants ou un large auditoire. 
- Ex. Cours d'histoire de la musique de Dujka Smoje, professeur honoraire de la Faculté de musique de l'Université de Montréal

- «Les sources de la musique occidentale (du Big Bang à Bach)»

- «Les grands moments de l'histoire musicale (de Bach à 1992)»

Voyons maintenant chacun de ces types de cours en étudiant l'exemple qui lui a été associé.

\section{Le cours magistral et sa version « à distance "}

\section{Description du cours d'histoire de la musique de Dujka Smoje de la Faculté de musique de l'Université de Montréal}

De prime abord, le cours d'histoire de la musique peut sembler être un des cours les plus faciles à intégrer dans un enseignement à distance, car l'on peut imaginer qu'il ne s'agit que d'enregistrer sur bande-vidéo le professeur qui donne son cours en classe et ensuite de le diffuser à la radio ou mieux, à la télévision.

Or le projet et sa démonstration peuvent être plus complexes. Nous évoquerons ici la version télévisuelle du cours d'histoire de la musique de Dujka Smoje, professeur honoraire de la Faculté de musique de l'Université de Montréal, qui montre toute l'exigence que la mise sur pied d'un tel enseignement à distance peut demander mais aussi les avantages que le programme révèle.

Ces deux cours créés respectivement en 1989 et en 1992 ont été une expérience innovatrice réalisée par Dujka Smoje et produite par la Faculté de musique avec l'aide technique de l'équipe de l'audiovisuel de l'Université de Montréal.

\section{Difficultés de réalisation}

De fait, Dujka Smoje aurait pu simplement demander au technicien à la caméra de filmer son cours afin de transmettre le contenu tel quel à ses étudiants qui pouvaient ensuite en disposer à leur guise. Mais Madame Smoje a préféré s'interroger sur le médium audiovisuel lui-même et elle a pensé à une méthode originale, inspirée du modèle platonicien, pour composer un cours inédit pour la télévision.

La stratégie adoptée pour passer à l'écran mon enseignement a été de renoncer au monologue - le professeur disant ordinairement le contenu de son cours comme il fait en classe - pour utiliser la manière du « dialogue ", c'est-à-dire en donnant son cours dans un échange arrangé avec un animateur et par le jeu de " questions et réponses " (selon le modèle platonicien) : ce moyen s'est avéré le plus efficace pour traduire mon enseignement. (Smoje 2008)

Les difficultés strictement pédagogiques ont été après coup de transposer le cours (monologue) en émission télé (dialogue) et de resserrer un contenu de cours de trois heures en une émission de 56 minutes sans que cette réduction dans le temps n'entraîne une simplification excessive du propos. La recherche de documentation visuelle et d'illustrations sonores appropriées et pouvant convenir au médium de la télévision a été le prochain défi pédagogique. Enfin, 
la structure de l'exposé a représenté la dernière problématique : en effet, il est connu que l'attention d'un spectateur se disperse assez rapidement, aussi Madame Smoje a choisi d'organiser son cours en séquences filmées de moins de 5 minutes chacune, permettant en cela aux spectateurs-étudiants de prévoir un comportement d'écoute assidue durant ce laps de temps défini.

\section{Les avantages pédagogiques de l'entreprise}

Concernant l'enseignement donné en classe aux étudiants en histoire de la musique, la possibilité de combiner le cours et les cassettes vidéo ont montré rapidement tout le bénéfice qu'on pouvait en tirer : en effet, aussitôt réalisées, les cassettes VHS ont servi d'outil pédagogique fondamental. L'essentiel de l'enseignement se trouvant sur ces cassettes, les étudiants pouvaient les réécouter autant de fois que nécessaire. De même, ils pouvaient fragmenter le contenu et préparer les questions qu'ils souhaitaient poser en classe la semaine suivante. De son côté, le professeur, sachant que les étudiants avaient visionné le cours à l'avance, pouvaient se permettre de développer et d'approfondir certains aspects du sujet du cours. Dujka Smoje relate elle-même :

Grâce aux scénarios des émissions, rigoureusement structurés et minutés, les cours étaient construits et articulés très scrupuleusement. L'exposé à l'écran était complété par les documents pédagogiques écrits, précisant les objectifs de chaque cours, identifiant le sujet de chaque séquence, les détails de l'illustration musicale, et le complément de lecture. Et, enfin, chaque fois que c'était possible, un extrait de la partition des œuvres était présenté. Ainsi l'étudiant avait une vision claire du sujet et de la démarche à suivre pour maîtriser la matière et la connaissance du répertoire. (Smoje 2008)

Ces vidéos du cours accompagnés des documents pédagogiques ont ainsi donné au professeur une très grande liberté d'innover en classe, d'avoir le temps de faire entendre davantage de musique du répertoire et de discuter des partitions comme de disposer de périodes pour les échanges suscités par les informations contenues dans la vidéo. À l'évidence, cette dynamique du cours télévisé de Madame Smoje correspond en tout point aux cours en ligne d'aujourd'hui avec leurs forums sur Internet et les rencontres en classe.

\section{Les droits d'exécution}

Dujka Smoje a utilisé ici le moyen préconisé aujourd'hui par différentes écoles de musique : en effet, lorsque c'est possible, il peut être demandé à une classe d'interprètes faisant partie de l'école où se donne le cours d'histoire de la musique, d'enregistrer des œuvres musicales choisies qui illustrent les exemples de la musique d'une époque et d'utiliser ces enregistrements avec l'accord des musiciens dans ce but pédagogique précis et à aucun autre escient. C'est ainsi qu'ont participé à la réalisation de ce cours de musique les professeurs de la Faculté accompagnés de leurs étudiants : Marc Durand, pianiste, Lise Daoust, flûtiste, Réjean Poirier, organiste et claveciniste, Alan Belkin, compositeur, Vladimir Landsman, violoniste, Yuli Turovsky, violoncelliste et chef d'orchestre ; 
le NEM, Nouvel Ensemble Moderne fondé et dirigé par Lorraine Vaillancourt, et I Musici, orchestre de 15 musiciens fondé et dirigé par Yuli Turovsky. Le problème des droits s'est arrêté avec la première période musicale définie par le cours, des origines à Jean-Sébastien Bach. Il est devenu omniprésent et presque insurmontable lorsque la seconde période, de Jean-Sébastien Bach à nos jours, a été abordée, à cause principalement des images. En effet, il a été quasi impossible d'utiliser des reproductions de peintures (par exemple celles de Henri Matisse), même si ces images n'étaient exposées que quelques secondes à l'écran, car les musées, et les agents qui les géraient, ont exigé à l'époque des sommes très importantes pour les ayants droit de chaque image. Il en a été de même pour la reproduction des exemples musicaux avec les sociétés de reproduction de musique.

Tenant compte qu'il était inséré plus de 800 images - reproductions d'œuvres d'art visuelles dans la deuxième série - , cela représentait une facture que l'Université n'était pas en mesure de payer. Les nombreuses discussions pour faire valoir le rôle éducatif de l'utilisation de ces reproductions, ainsi que toutes les explications montrant que rien ni personne - ni le professeur, ni l'institution - ne tiraient aucun avantage financier de ces cours, n'ont résolu le problème et les cours ont été retirés du circuit public. Ces difficultés (des droits) existaient en 1999, pour la diffusion des cours par la télévision, et aujourd'hui nous pouvons penser que la situation est inchangée. (Smoje 2008)

En effet, il semble qu'en 1992 les musées et les gestionnaires mandatés, pour faire respecter les ayants droit, aient été inflexibles. Pourtant, la contribution de ces travaux d'enseignement télévisuels à faire connaître et partager la culture des musées par un plus large public était évidente. Néanmoins l'idée de profit, dans les musées, a persisté au détriment de l'idée d'un enrichissement de l'esprit, contrairement aux professeurs et chercheurs impliqués dans le projet d'enseignement qui n'envisageaient tirer aucun bénéfice personnel au départ. Ce type d'entreprise, à la longue, devrait certainement mettre en place des droits pour les auteurs des cours et des illustrations sonores comme visuelles, mais ce n'est pas la motivation première des acteurs de ce projet d'enseignement. De plus, l'expérience montre que, souvent, c'est le contraire qui se produit : les enseignants qui, comme Madame Smoje, poursuivent des recherches dans le domaine de l'iconographie musicale, doivent nécessairement payer des droits pour chaque reproduction dans un article publié. Les auteurs doivent " ruser » en découpant les images et en reproduisant des fragments en blanc et noir pour réduire les coûts. Pourtant, généralement, ces mêmes auteurs ne perçoivent aucune compensation des revues qui les publient.

\section{Coûts de réalisation des cours à distance de l'histoire de la musique et frais d'inscription à ces cours pour les étudiants}

En ce qui a trait au coût de réalisation des cours à distance et des frais d'inscription à ces cours, Dujka Smoje suggère de considérer l'entreprise à moyen et à long terme. Les droits d'inscription ne peuvent pas compenser le coût de 
l'affaire dès la première année. Cependant, l'entreprise est parfaitement possible et rentable si ces frais sont répartis sur plusieurs trimestres, voire sur trois ou quatre ans, en mettant à jour régulièrement les documents pédagogiques en ligne, comme le permet actuellement l'usage des modèles (logiciels) de chaînes éditoriales ${ }^{15}$ tel que Opale de Scenari.

Le prix (de l'enseignement à distance) pourrait être légèrement supérieur par rapport à celui facturé par crédit des cours en classe. Au terme de deux ou trois ans, l'investissement peut être amorti. (Smoje 2008)

Autrefois, lorsque Dujka Smoje a conçu ses cours d'histoire télévisés et les documents pédagogiques qui les accompagnaient, Internet n'existait pas encore. Mais avec les moyens actuels, la conception d'un cours vidéo pour l'enseignement à distance serait tout à fait dynamique grâce à son complément en ligne. La question qui pourrait être posée à propos de l'enseignement à distance concerne l'intérêt du grand public.

L'Université n'a pas seulement la mission de former des professionnels, mais aussi d'ouvrir l'accès à la culture au grand public. De cette façon, les activités musicales pourraient compter sur un public élargi. Cette mission culturelle est souvent ignorée par l'université comme étant marginale et sans intérêt financier. Pourtant, les divers témoignages des personnes qui suivaient, en 1992, les cours d'Histoire de la musique à la télévision, ont souligné l'intérêt de ce type de solution. (Smoje 2008)

\section{Le cours en groupe moyen}

\section{Description du cours d'ethnomusicologie de Monique Desroches de la Fa- culté de musique de l'Université de Montréal}

À l'Université de Montréal, par l'entremise de SUITE-CEFES et avec l'aide de techniciens de la plateforme WebCT, un cours d'ethnomusicologie a été réalisé par le professeur Monique Desroches, Responsable du secteur Ethnomusicologie de la Faculté de musique. Monique Desroches a conçu le cours «Introduction à l'ethnomusicologie (cours en ligne) " (MUL 3314). La réalisation comme la production ont nécessité la collaboration d'un technicien de WebCT durant deux ans, à raison d'une rencontre par semaine. De même, Madame Desroches a été invitée à suivre une formation de 45 heures pour connaître et apprendre le fonctionnement de la plateforme WebCT. Cette entreprise a permis de construire un cours, annoncé dans le site du Laboratoire LRMM - Laboratoire de Recherche des Musiques du Monde - qui s'adresse aux étudiants qui peuvent s'y inscrire au même titre qu'un cours régulier de la programmation des cours de la Faculté de musique. À l'inverse du cours d'histoire de Dujka Smoje, Monique Desroches incite ses étudiants à assister en classe à son enseignement, puis à aller suivre le programme complémentaire de son cours sur le

15 Une chaîne éditoriale est un moyen méthodologique et technologique développé par la recherche en ingénierie documentaire. Le procédé consiste à réaliser un modèle de document, à assister les tâches de création du contenu et à automatiser leur mise en forme. 
module en ligne. Des discussions sur forums hebdomadaires sont assurées et les étudiants sont conviés à y participer régulièrement.

Concernant la contribution et les avantages pédagogiques de ce cours sur Internet, Desroches souligne le dynamisme (cette qualité fréquemment énoncée dans l'ensemble des valeurs qu'on attribue à l'e-learning) dans la participation des étudiants à ses cours. De même que le professeur signale la grande flexibilité dans la présentation du cours et la quantité importante d'informations qu'elle peut joindre à ses documents pédagogiques sous forme d'images, d'illustrations sonores et de vidéo de toutes sortes. En effet, l'ethnomusicologie est connue pour être essentiellement un travail qui repose sur la collecte de sources inédites de musiques populaires ou classiques de toutes régions du globe ; son activité essentielle est souvent de réunir dans les meilleures conditions les exemples musicaux trouvés et choisis des civilisations étudiées. Par conséquent, la question des droits d'auteur, des compositeurs ou d'exécution est plus facilement esquivée qu'en ce qui a trait à la musique classique parce qu'il s'agit souvent d'œuvres non encore répertoriées et que ce premier exercice de catalogage permet de classer et conserver souvent ces musiques pour la première fois et de profiter de cette occasion pour signifier et protéger les droits des musiciens qui les interprètent.

\section{Description du cours d'harmonie tonale de Josée April du Conservatoire de musique du Québec à Rimouski}

Le cours d'harmonie tonale de Josée April, Mo4-201-202-203-204, est actuellement en travail de réalisation à distance par le professeur et par l'auteur du présent texte : en effet, il est entièrement enregistré sur vidéos numériques, de la façon dont il est donné en classe au Conservatoire de musique de Rimouski. Le cours fait l'objet de l'enregistrement de trois disquettes vidéo de 20 minutes chacune, totalisant l'heure de la leçon, l'ensemble du cours se divisant sur deux sessions de 14 leçons respectives. Ces vidéos servent ensuite de matériel de base qui donne le contenu théorique et pratique de la leçon. Par exemple, on voit Josée April expliciter les règles d'harmonie en utilisant un exemple d'une mélodie à harmoniser inscrite au tableau. Une fois données, les explications orales sont ajoutées à la vidéo sous forme de sous-titres. Ces sous-titres sont ensuite repris intégralement pour être rassemblés par écrits dans les pages qui accompagnent en parallèle le déroulement de la vidéo : ainsi les étudiants peuvent-ils suivre en lecture les mêmes explications qui sont données oralement par April. À la fin de chaque leçon, on retrouve deux exercices à réaliser à la maison, tels qu'il est demandé en classe et la correction de ces exercices se retrouve en début de leçon suivante en vidéo, c'est-à-dire la leçon suivante en classe. L'harmonisation d'une mélodie étant un travail qui n'est pas accompli de la même façon par chaque étudiant, car il s'agit d'une création musicale originale, la correction de chaque devoir est individuelle : cela signifie que le devoir réalisé par un étudiant qui suit le cours à chaque semaine sur Internet doit pouvoir être rendu au professeur ou à un tuteur avant la prochaine leçon.

La notion de tutorat, ou d'accompagnement d'un élève suivant un cours à distance, est une conception qui a été développée depuis l'origine de 
l'enseignement à distance : il s'agit de mettre en place une équipe de correcteurs, ou de «soutien». En effet, traditionnellement un professeur donnait son cours à une douzaine d'étudiants. À cela correspondait bien normalement une douzaine de copies à corriger. Mais si un cours est diffusé à distance à 50 ou 100 élèves, il devient impossible pour le professeur qui a conçu le cours - et qui a déjà eu un travail supplémentaire pour ce faire - de corriger autant de copies qu'il y a d'élèves. Cela crée l'obligation d'engager des tuteurs qui peuvent être des étudiants finissant. Concernant les droits d'auteurs des musiques, la question s'efface automatiquement puisque les étudiants sont eux-mêmes les compositeurs des harmonies.

\section{Le cours privé ou le cours en petit groupe}

\section{Description du cours de saxophone de Benoît Plourde du Conservatoire de musique du Québec à Rimouski}

Enfin, une expérience de cours à distance d'un instrument de musique, du professeur à son élève, a été tentée par Benoît Plourde, Directeur du Conservatoire de musique du Québec à Rimouski, professeur de saxophone. Cette expérience a été enregistrée par Josée April sur vidéo numérique. D’autres cours d'interprétation ont été explorés dans cette institution, tels que l'enseignement du piano et du clavecin, mais notre choix s'est arrêté sur l'instrument à vent à cause des difficultés spécifiques que son enseignement soulevait. En effet, le saxophone est un instrument que l'on porte à la bouche pour jouer. On introduit d'abord une pièce que l'on appelle une anche simple immédiatement sur l'embouchure; cette anche se trouve au contact direct des lèvres lorsqu'on en joue. Or dans l'enseignement, la façon de placer les lèvres sur cette partie doit être montrée sur plusieurs côtés du visage : de profil et de face. Vouloir apercevoir ces deux perspectives entraîne de filmer successivement les deux scènes, puis d'utiliser la technique cinématographique de "l'écran divisé » avec laquelle on peut afficher les deux perspectives simultanément. C'est une difficulté mineure, qui peut être aisément surmontée, mais qui manifeste la complexité de l'approche lorsqu'il s'agit de montrer comment pratiquer un instrument sans que le maître ne se trouve dans la même pièce que son étudiant. Par ailleurs, depuis une vingtaine d'années, il est obligatoire en Amérique du Nord d'éviter tout contact physique non nécessaire d'un maître sur son élève, à cause de plaintes concernant les contacts qui pourraient être interprétés comme étant trop intimes. Cette nouvelle contrainte astreint le professeur à développer un langage pédagogique explicite et imagé pour que son étudiant comprenne sans qu'il soit nécessaire de porter la main à son visage ou sur son bras. Cette exigence comporte heureusement des bienfaits : le vocabulaire s'est ainsi diversifié, nuancé et élaboré en profondeur et l'étudiant s'exprime désormais au sujet de son instrument avec beaucoup de clarté et de compétence. L'inconvénient qui, néanmoins, est rapporté dans l'expérience de l'enseignement à distance du jeu d'instrument concerne la maturité de l'étudiant : par exemple, un enfant de douze ans ne saura pas profiter pleinement de ce type d'enseignement; il a besoin de la présence physique de son professeur dans la même salle que 
lui, d'entendre sa voix à ses côtés, et de ressentir sa présence. Sinon, la qualité d'enregistrement sonore est amplement suffisante pour que l'élève ait une idée précise de la sonorité qu’on attend de lui dans le jeu de son interprétation, comme les prises de vue par la caméra des partitions et des feuilles de musique permettent une lecture claire et il est même facile de voir les annotations ajoutées au crayon dans le cahier du maître pour l'étudiant. Cela dit, nous constatons que ce cours sur vidéo est montré sans que le spectateur puisse participer. Cependant, on peut aussi faire ce cours à distance par le biais de la WebCamera et faire ainsi un cours d'interprétation « en public » à distance. Toutefois, l'exemple suivant montre que, dans le cours à distance de piano réalisé par le Département de musique de l'Université d'Ottawa, la vidéo peut être employée de façon «interactive » par les réalisateurs comme par les spectateurs.

\section{Description du cours de piano à distance et la "technologie d'annotation de la vidéo " du Département de musique de l'Université d'Ottawa}

La vidéo est utilisée pour soutenir l'apprentissage et l'enseignement dans plusieurs domaines. Cependant, la majorité des applications de la vidéo à des fins d'apprentissage et d'enseignement utilise peu les avantages de la vidéo numérique. Un élément fondamental associé à la vidéo numérique est la possibilité d'annoter de manière automatique ou manuelle le contenu visuel et sonore et d'amalgamer ces annotations à la vidéo pour créer de nouveaux documents multimédias. (Edmond et al 2006, p. 49)

Malgré l'apparente difficulté de traduire un cours d'interprétation musicale pour l'enseignement à distance sur Internet, le Département de musique de l'Université d'Ottawa expérimente depuis des années, avec l'Institut de technologie de l'information, le Conseil national de recherches Canada et le Département des sciences de l'éducation de l'Université du Québec en Outaouais, l'enseignement à distance du piano avec l'aide d'enregistrements vidéos. Cette expérimentation porte le nom de « Développement de programmes informatiques et de logiciels qui procureront l'interface technique essentielle et les outils pédagogiques pour l'enseignement du piano à distance ». Il est dit à propos de la vidéo employée simplement comme " témoin » d'un cours d'interprétation :

Sans des moyens de naviguer dans un document audiovisuel, celui-ci demeure essentiellement un médium narratif qui supporte difficilement un apprentissage actif où l'apprenant a un contrôle sur le médium (Laurillard, 1995 ; Shephard, 2003).(Edmond et al 2006, p. 50)

En effet, l'utilisation de la technologie d'annotation vidéo est l'élément clé dans ce laboratoire : cette technique permet d'ajouter des commentaires de formation au fur et à mesure du déroulement de la vidéo. Ces annotations sont aussi un moyen de "naviguer » dans le document visuel et d'atteindre directement des sujets précis de l'apprentissage qui intéressent l'enseignant ou l'étudiant. 
L'ajout d'annotations ouvre toutes sortes de possibilités sur le plan du développement des ressources médiatiques pour soutenir l'apprentissage, l'enseignement et la recherche sur la pédagogie du piano. Ainsi, l'indexation de leçons de piano, en y ajoutant des annotations, permet d'accéder à des segments particuliers de vidéos à partir de divers critères de recherche comme le type d'intervention pédagogique, une séquence de notes jouée au piano, le geste technique, ou même la qualité expressive du jeu musical. (Edmond et al 2006, p. 56)

Ces recherches pour l'enseignement à distance du piano ne fonctionnent pas selon les TICE (Technologies de l'information et de la communication pour l'éducation) ou TICCE (Technologies de l'information, la communication et la création pour l'enseignement) qui sont assujettis au comité de normalisation JTC1SC-36 proposant la norme SCORM dont le rôle est de mettre en forme le document-texte du cours et d'harmoniser le fonctionnement de ses éléments constituants pour le rendre inter-opérable. Ces documents textes comportent des fichiers d'images comme des fichiers sonores, ou des fichiers vidéos mais ces fichiers sonores et visuels n'entrent pas dans la composition du document SCORM en tant que tel : les documents d'enseignement normalisés SCORM peuvent contenir des fichiers audiovisuels sans pour autant avoir les capacités de gérer leurs métadonnées. De leur côté, les vidéos font partie du domaine de MPEG, Moving Picture Experts Group, qui est le groupe de travail SC29/WG11 du comité technique mixte JTC1 de l'ISO et de la CEI pour les technologies de l'information. Ce groupe d'experts est chargé du développement des normes internationales pour la compression, la décompression, le traitement et le codage de la vidéo, de l'audio et de leur combinaison, de façon à satisfaire une large gamme d'applications.

\section{Les normes MPEG explicitées par Alain Vaucelle, chercheur du département Artémis Telecom Paris :}

Les normes audiovisuelles employées sont MPEG-1 \& 2, MPEG-4, MPEG-7 et MPEG-21 dont les définitions et les précisions sont apportées ici par Alain Vaucelle. MPEG-1 \& 2 exécute la compression de l'information et représente la norme de codage de l'audio et de la vidéo pour le transport pour la télévision numérique. Puis MPEG-4 propose l'organisation des normes d'un audiovisuel et d'un multimédia très exhaustivement interactif et intégralement structuré selon les principes des langages à balises.

Un flux MPEG-4 est un contenu vidéo enrichi de divers éléments d'informations relatifs aux différents objets individuels considérés, comme durée de vie, régions support, emplacement dans une scène... Il vient tout naturellement à l'esprit la possibilité d'enrichir encore davantage cette représentation, en associant aux différents objets des descripteurs spécifiques débouchant sur des fonctionnalités nouvelles, comme par exemple l'Accès automatique et les requêtes par le contenu. C'est l'objet de MPEG-7 (Multimédia Content Description Interface). (Vaucelle 2010) 
MPEG-7 est l'organisation des normes documentaires du domaine. Norme ISO/IEC 15938, 2002 édifie une palette d'outils normalisés pour indexer et décrire syntaxiquement de façon automatique ou semi-automatique tout contenu multimédia. Une même information pourra être traitée en fonction des capacités communicationnelles recherchées, allant du spatio-temporel (audio et vidéo traités séparément) à une description sémantique du flux de données.

MPEG-7 peut être associé aux autres descripteurs spécifiant le format, les conditions d'accès, leurs classifications, les liens pertinents en relation avec l'information initiale, le contexte d'enregistrement ou de la diffusion du matériel : c'est la possibilité de naviguer, de chercher, de filtrer et de s'approprier l'information dans n'importe quel corpus multimédia ouvert. (Vaucelle 2010)

Et, pour terminer, $M P E G$-21 qui distingue l'organisation des normes de l'intégration des services dans la totalité du domaine multimédia (ce qui implique la création notamment d'une couche du e-procurement (approvisionnement électronique), notamment lorsque le multimédia devient interactif).

MPEG-21 (ISO/IEC 21000. 2003), appelé Multimedia Framework, se propose notamment de lever ce verrou technologique en standardisant des descriptions non seulement des contenus, mais aussi de tous les éléments susceptibles d'intervenir dans la chaîne de consommation, depuis la création, en passant par la diffusion et en allant jusqu'à l'utilisateur final. (Vaucelle 2010)

Dans le laboratoire du cours de piano à distance de l'Université d'Ottawa, l'utilisation du protocole de communication MIDI est directement reliée au travail d'annotation de la vidéo. L'annotation multimodale permet aussi d'associer le contenu MIDI au document audiovisuel, permettant ainsi de faire jouer le piano simultanément avec le visionnement ou même d'utiliser le piano comme outil de recherche de documents vidéo (comme rechercher tous les documents vidéo qui incluent une certaine séquence de notes). Les normes et standards MPEG gèrent les métadonnées qui servent à fabriquer des contenus audiovisuels inter-opérables permettant de bâtir le cours qu'il est possible ensuite de diffuser sur Internet. Cet enseignement à distance « vidéo » du piano est un cours fonctionnant sur le modèle des émissions de la télévision numérique, non des programmes d'enseignement à distance ou d'e-learning traditionnel.

\section{Conclusion}

L'e-learning en général développe l'idée et l'approche pédagogique « d'autoformation » qui sollicite une conduite autonome de l'étudiant dans les différentes étapes et niveaux de son apprentissage. Cette attitude en formation musicale est parfaitement viable dans de nombreux types de cours, en commençant avec la classe magistrale (cours d'histoire) jusqu'à la classe en privé (cours de piano ou d'un autre instrument de musique). Ont été distingués les cours d'autoformation (les logiciels de reconnaissance auditive, de dictées et d'analyse musicales) des cours traditionnels mis à distance (cours d'harmonie tonale, d'ethnomusicologie, d'histoire de la musique et les cours d'interprétation musicale). Cette 
distinction montre que plusieurs réalisations de cours ont connu un succès immédiat auprès des étudiants, des professeurs et des directeurs des écoles de musique. En aucun cas la nécessité du contact avec un professeur ou un tuteur n'est remise en question : seul le mode de fréquentation est différent, ce qui ne veut pas dire que le nombre de fréquentations doive baisser. Qu'ils se passent en visioconférence ou sur forums, les échanges entre professeurs et étudiants, comme entre les apprenants eux-mêmes, sont encouragés et peut-être plus motivés, car l'élève est davantage maître de son apprentissage et doit s'impliquer dans les modalités et le parcours de sa formation.

À propos des droits d'auteurs, d'exécution et de reproduction, il existe plusieurs manières aujourd'hui de contourner le problème. Cependant, les solutions proposées sont peu satisfaisantes au regard de droits et de règlements en cause. Ce dysfonctionnement fait appel à de nouvelles perspectives que le « tout numérique " permet d'entrevoir, comme les révolutions accomplies dans le domaine de la télévision numérique et ce que ces révolutions laissent présager.

Depuis février 2010, la télévision numérique a été implantée en région d'Alsace comme base d'expérimentation de la généralisation de la télévision numérique en France et la diffusion de la télévision analogique par voie terrestre cessera pour les trois quarts de la population française le 30 novembre 2011. Aux États-Unis, l'Advanced Television Systems Committee (ATSC) est le groupe qui a contribué au développement du nouveau standard de télévision numérique. Ce standard a été adopté par le Canada, le Mexique et la Corée du Sud. Il doit remplacer le système analogique NTSC (National Television System Committee), standard de codage analogique de la vidéo en couleurs qui opérait depuis 1953. La télévision numérique commence avec le développement en audiovisuel de la norme MPEG (Moving Picture Experts Group) et ses déclinaisons (MPEG-4, MPEG-7 et MPEG-21). Ces normes MPEG favorisent la compression de l'information (MPEG-1 et 2), l'usage des applications multimédias et la description d'un codage vidéo (MPEG-4), l'indexation et la recherche de documents multimédia (MPEG-7) et la spécification d'une architecture permettant l'interopérabilité et l'utilisation transparente des représentations audiovisuelles numériques et la gestion automatique des droits numériques (MPEG-21). Le monde de l'audiovisuel maîtrise ainsi déjà le problème des droits, quels qu'ils soient (d'auteur, d'exécution, de production) depuis le standard MPEG-4 et définitivement avec la norme MPEG-21. Cette dernière gestion qui est automatique peut être comparée à «l'achat d'un billet d'avion sur Internet». En effet, la métaphore du billet d'avion « acheté sur Internet pour aller d'une grande ville à un village éloigné » est celle qui exprime le mieux la complexité du problème et l'efficacité de sa solution : d'un simple clic, l'ordre est donné à l'agent de voyage qui apportera un résultat au bout d'environ cinq minutes. Dès lors, le voyage, son itinéraire en avion, suivi du trajet en train puis en bus, jusqu'à la réservation de l'hôtel, est prévu : toutes les variantes des coûts entre les pays et entre les moyens de transport ont été analysées, répertoriées et exprimées dans une somme finale proposée à l'acheteur. C'est ainsi que les droits des images, des textes, des musiques, etc., sont entièrement pris en charge par la norme MPEG21 dans le cadre d'une émission numérique audiovisuelle aujourd'hui. 
Les TICE réorganisent la circulation des savoirs pour l'enseignement et la formation professionnelle. La réussite de l'accès à ces contenus et de la maîtrise de ces technologies vers le plus grand nombre est étroitement dépendante de leur normalisation. Les instances de normalisation tentent de couvrir les spécifications inhérentes aux TICE afin de rendre possible cette convergence des médias. Cette convergence questionne la notion même de document. (Vaucelle 2009, p. 15)

Ainsi s'exprime Alain Vaucelle à propos des TICE. Il suggère une « recréation de la notion de document » puisque désormais le terme "document » englobe des informations de toute provenance : textes, images, vidéos, sons, animations. Alain Vaucelle et Henri Hudrisier, Expert du Comité de normalisation SC-36, Maître de conférences et $\mathrm{HDR}^{16}$ à l'Université de Paris 8, proposent que des passerelles entre les domaines de l'audiovisuel numérique et de l'e-learning soient établies afin que chacun des deux champs d'activité soient enrichis des découvertes et des solutions trouvées par l'autre.

Pour normaliser ces technologiques de l'éducation et de la formation, il est nécessaire de parvenir à des consensus favorisant la médiation entre des pédagogues et des communautés d'experts en multimédia. L'intérêt de la norme MPEG-21 est de définir un cadre normatif qui couvre l'ensemble de la chaîne de production et de distribution des ressources multimédias. Face à ces enjeux, on doit analyser à travers la famille MPEG, les bénéfices de cette approche normative pour les métadonnées associées aux TICE et au multimédia. (Vaucelle 2009, p. 15)

Ces deux domaines peuvent s'apporter mutuellement lorsqu'ils gèrent des contenus numériques similaires - textes, vidéos, images, etc. - selon des normes respectives, mais non hermétiques. Par exemple, il est possible que l'e-learning emprunte des réponses trouvées par l'audiovisuel numérique pour régler des problèmes identiques, et réciproquement, si, éventuellement, le monde de l'audiovisuel décidait de produire de l'enseignement à grande échelle sur des chaînes télévisées. Pour terminer ce rapide parcours de l'enseignement à distance de la musique et des outils qui permettent aujourd'hui de traduire ces cours « en ligne ", nous rappellerons qu'il existe nombre d'autres expériences, que celles mentionnées ici, en e-learning musical ou formation à distance de la musique dans divers établissements d'enseignement. Et les expérimentations vont en se propageant : à la suite de l'observation du succès de l'e-learning musical dans les institutions canadiennes, André Picard, Directeur des études et du développement des Conservatoires de musique et d'art dramatique du Québec, a annoncé l'entreprise, depuis février 2010, pour la création d'une plateforme de formation en ligne mettant en réseau l'ensemble des sept Conservatoires du Québec; il s'agissait de proposer graduellement des cours à distance inédits à ses étudiants. Car la réalisation de cours de musique à distance propose une aussi grande variété de productions qu'il existe une diversité et pluralité des enseignements caractérisés par les établissements dont ils sont issus - Facultés

16 HDR est le sigle désignant l'« Habilitation à diriger des recherches ». 
d'universités qui enseignent la pratique instrumentale et vocale tout en se spécialisant dans la recherche, Conservatoires qui se destinent principalement à l'interprétation instrumentale et vocale ou les diverses écoles qui favorisent certaines activités musicales. Cette abondance est au cœur du patrimoine pédagogique que les grands maîtres de musique de par le monde représentent. Il serait souhaitable de pouvoir en répertorier le plus d'exemples possibles qui témoigneraient de manière éloquente de la multiformité de l'enseignement musical, devenu quasi protéiforme lorsqu'il est adapté dorénavant «à distance ».

\section{RÉFÉRENCES}

Baron, Georges-Louis et Éric Dane. 2009. "Technologies éducatives et francophonie : un champ de recherches pluriel ». La recherche en technologie éducative: Un guide pour découvrir un domaine en émergence, sous la dir. de Christian Depover, 25-33. Paris : Agence Universitaire de la Francophonie / Éditions des archives contemporaines.

Battier, Marc. 2003. "Science et technologie comme sources d'inspiration ". Musiques. Une Encyclopédie pour le XXI siècle, "1. Musiques du XXe siècle », sous la dir. de Jean-Jacques Nattiez, 512-532. Arles-Paris : Actes Sud/ Cité de la musique.

Ben Henda, Mokhtar, Henri Hudrisier, Françoise Preteux et Alain Vaucelle. 2008. «Les normes des TICE du XXIème siècle ». Colloque International de l'Université de l'Ère Numérique, Bordeaux. 10-11-12 décembre 2008 : 1-10.

Bezard, Michel. 2003. "TICCE : le MIDI à l'heure du second C ». Les dossiers de l'ingénierie éducative: Des outils pour la musique, $\mathrm{n}^{\circ} 43: 1-71$.

Brunswick, Étienne. 1970. "Hier, l'audiovisuel : demain, la technologie de l'éducation ». Media, $\mathrm{n}^{\circ} 18: 15-23$.

Dauphin, Claude. 2004. «Les grandes méthodes pédagogiques du XX $\mathrm{X}^{\mathrm{e}}$ siècle ». Musiques. Une Encyclopédie pour le XXI siècle, «2. Les savoirs musicaux », sous la dir. de Jean-Jacques Nattiez, 833-853. Arles-Paris : Actes Sud/Cité de la musique.

Delalande, François. 2003. "D’une technologie à l'autre ». Les dossiers de l'ingénierie éducative: Des outils pour la musique, $\mathrm{n}^{\circ}$ 43: 6-10.

Dubeau, Annie et Véronique Besançon. 2005. "Environnement WebCT : des outils pour enseigner et apprendre ». Les Dossiers technopédagogiques de Profetic. En ligne : http://www.profetic.org/dossiers/spip.php?rubrique107, [consulté le 20 juillet 2009].

Edmond, Bruno, Marion Barfurth, Gilles Comeau et Martin Brooks. 2006. « Technologies d'annotation vidéo et leurs applications à la pédagogie du piano ». Recherche en éducation musicale, $\mathrm{n}^{\circ} 24: 49-60$.

Finkielktraut, Alain. 2007. La querelle de l'école. Paris : Stock.

Fouilloux, Jean-Martial. 2003. "Découvrir le pouvoir du son dans l'œuvre audiovisuelle ». Les dossiers de l'ingénierie éducative : Des outils pour la musique, $\mathrm{n}^{\circ} 43: 34-35$.

Gégout, Étienne. 2003. "Les grandes familles logicielles ». Les dossiers de l'ingénierie éducative: Des outils pour la musique, $\mathrm{n}^{\circ} 43: 46-47$. 
2003. "Créer une pièce vocale sur l'exemple de Nuits de Iannis Xenakis ». Les dossiers de l'ingénierie éducative: Des outils pour la musique, $\mathrm{n}^{\circ} 43: 31$. Gégout, Étienne et Christophe Przybylski. 2003. "Trois outils indispensables :1. Cubase, au service des pratiques vocales. 2. MusiqueLab, pour explorer et manipuler les matériaux de la musique. 3. Asymétrix Présentation, pour l'écoute interactive ». Les dossiers de l'ingénierie éducative : Des outils pour la musique, $\mathrm{n}^{\circ} 43: 48-55$.

Hudrisier, Henri. 2004. "E-learning et normalisation, définitions, enjeux et contexte ». Enseignement ouvert et à distance : Épistémologie et usages, sous la dir. d'Imad Saleh et Soufiane Bouyahi, 203-225. Paris : Lavoisier - Hermès.

Jacquinot, Geneviève. 1985. L'école devant les écrans. Paris : ESF. Jaillet, Alain. 2004. L'école à l'ère numérique. Paris : L'Harmattan.

Karsenti, Thierry, Vassili Komis et Christian Depover. 2009. « Les nouveaux outils et les nouvelles pratiques de recherche issus des technologies de l'information et de la communication ". La recherche en technologie éducative : Un guide pour découvrir un domaine en émergence, sous la dir. de Christian Depover, 35-55. Paris : Agence Universitaire de la Francophonie / Éditions des archives contemporaines.

Lacasse, Serge. 2010. Entretien accordé à l'auteur, le 28 janvier.

Lebrun, Marcel. 2002. Des technologies pour enseigner et apprendre. Bruxelles : De Boeck.

Lemeu, Michel. 2003. "Des ressources : CD audio, CD-Rom, DVD, sites internet ». Les dossiers de l'ingénierie éducative : Des outils pour la musique, $\mathrm{n}^{\circ} 43: 54-60$.

Maestricci, Vincent. 2003. "Demain, l'éducation musicale ». Les dossiers de l'ingénierie éducative: Des outils pour la musique, $\mathrm{n}^{\circ} 43: 2-5$.

- 2003. "Les technologies et les programmes d'enseignement ». Les dossiers de l'ingénierie éducative : Des outils pour la musique, $\mathrm{n}^{\circ} 43: 18-20$.

Marfaing, Daniel. 2003. "La création pour l'écoute, l'écoute pour la création ». Les dossiers de l'ingénierie éducative: Des outils pour la musique, $\mathrm{n}^{\circ} 43$ : 27-30.

Maurer, Jean-Marc. 2003. "Cartable électronique et éducation musicale ». Les dossiers de l'ingénierie éducative: Des outils pour la musique, $\mathrm{n}^{\circ} 43: 39-41$.

Moeglin, Pierre. 1999. Du mode d'existence des outils pour apprendre. En ligne : http://w3.u-grenoble3.fr/les_enjeux/200o/Moeglin/home.html, [consulté le 18 juin 2009].

Plas, Colette. 2003. " Une politique publique de développement d'outils technologiques pour l'éducation musicale ». Les dossiers de l'ingénierie éducative : Des outils pour la musique, $\mathrm{n}^{\circ} 43: 42-44$.

Poisson, Philippe. 2003. "Les TICCE au service de la transversalité - pistes vers d'autres disciplines ». Les dossiers de l'ingénierie éducative : Des outils pour la musique, $\mathrm{n}^{\circ} 43: 36-38$.

Saleh, Imad et Soufiane Bouyahi. 2004. «Les TIC dans les processus d'apprentissages ». Enseignement ouvert et à distance : Épistémologie et usages, sous la dir. d'Imad Saleh et Soufiane Bouyahi, 19-33. Paris : Lavoisier - Hermès. 
Smoje, Dujka. 2008. Entretien accordé à l'auteur, le 12 décembre.

Stiegler, Bernard. 2003. "Bouillonnements organologiques et enseignement musical ». Les dossiers de l'ingénierie éducative: Des outils pour la musique, $n^{\circ} 43: 11-15$.

Vaucelle, Alain. 2009. "MPEG-21 : la norme des TICE du XXIème siècle? ». Hermès. Rétrospective et perspective 1989-2009 Hypertext \& Hypermedia, Products, Tools and Methods (Actes du colloque), 115-121. Paris : Université Paris 8.

-2010. Entretien avec l'auteur, le $1^{\mathrm{er}}$ février.

\title{
RÉSUMÉ
}

Aujourd'hui l'e-learning musical est un outil d'apprentissage de la musique en plein essor depuis le développement des technologies tel que le standard MIDI - permettant la communication entre ordinateurs et instruments de musique -, Internet, le Web et les multimédias. Tous ces domaines reliés favorisent la création de cours mis sur logiciels qui peuvent être diffusés sur Internet ou en Intranet dans les écoles. Cette avancée dans l'éducation musicale se constate partout dans le monde et nous verrons dans cet article différents exemples et procédés d'apprentissage à distance de la musique qui sont mis en œuvre en France et au Canada.

\begin{abstract}
Musical e-learning is nowadays possible since the development of the technologies as the standard MIDI, which allow communication between computers and musical instruments, Internet, Web and multimedia tools. All those components combined together help to produce musical courses on CDs, which can be brought after on Internet or Intranet of musical institutions. This progress in musical education can be observed all over the world and we will see in this article various examples of musical's e-learning which as been applied in schools in France and Canada.
\end{abstract}

